\title{
Association Between Sleep Quality and Semen Parameters and Reproductive Hormones: A Cross-Sectional Study in Zhejiang, China
}

This article was published in the following Dove Press journal: Nature and Science of Sleep

\section{Cong-Qi Du (D) \\ Yong-Yi Yang ${ }^{2}$ \\ Jing Chen' \\ Lei Feng ${ }^{3}$ \\ Wen-Qin Lin'}

'Reproductive Medicine Center, The First Affiliated Hospital, College of Medicine, Zhejiang University, Hangzhou, People's Republic of China; ${ }^{2}$ Department of Gynaecology, The First Affiliated Hospital, College of Medicine, Zhejiang University, Hangzhou, People's Republic of China; ${ }^{3}$ Information Center, The First Affiliated Hospital, College of Medicine, Zhejiang University, Hangzhou, People's Republic of China
Correspondence: Wen-Qin Lin The First Affiliated Hospital, College of Medicine, Zhejiang University, Hangzhou 310003, People's Republic of China Emailwzlwq70@I26.com
Background: The effects of sleep duration on semen quality have been documented in many epidemiological studies. However, the association between sleep quality and semen parameters and reproductive hormones is still unclear.

Patients Enrollment and Methods: We conducted a cross-sectional study among 970 outpatients from the Reproductive Medicine Center in Zhejiang, China between October 2017 and July 2019. All participants delivered a semen sample, underwent a physical examination, and answered a questionnaire to provide the following information: demographics, life habits, and sleep habits. Sleep quality was measured using the Pittsburgh Sleep Quality Index (PSQI). We first divided the patients into two groups according to sleep quality (good sleep: PQSI $<5$ and poor sleep: PSQI $\geq 5$ ). Then, we analyzed routine sperm parameters (semen volume, sperm total motility, progressive motility, sperm concentration, total sperm number, and normal sperm morphology) and reproductive hormones (folliclestimulating hormone, luteinizing hormone, estrogen, testosterone, and prolactin) of each group. Finally, we used multivariate linear regression analysis and Spearman correlation coefficients to examine the relationship between sleep quality (discrete variable or dichotomous variable) and sperm parameters, reproductive hormones.

Results: A negative correlation was found between the general PSQI scores and several semen parameters: total motility $(\mathrm{r}=-0.187979, p<0.001)$, progressive motility $(\mathrm{r}=-0.192902, p<0.001)$, concentration $(\mathrm{r}=-0.167063, p<0.001)$, total sperm number $(\mathrm{r}=-0.160008, p<0.001)$, and normal sperm morphology $(\mathrm{r}=-0.124511, p<0.001)$. However, there was no significant correlation between the semen volume, all reproductive hormones and the general PSQI scores. After adjusting for confounders, men with poor sleep had lower total motility $(\beta=-9.287 ; 95 \% \mathrm{CI},-12.050,-6.523)$, progressive motility $(\beta=-8.853 ; 95 \% \mathrm{CI},-11.526,-6.180)$, concentration (log scale, $\beta=-0.131 ; 95 \% \mathrm{CI}$, $-0.181,-0.082$ ), total sperm number ( $\log$ scale, $\beta=-0.137 ; 95 \% \mathrm{CI},-0.189,-0.084)$, and normal sperm morphology $(\beta=-1.195 ; 95 \% \mathrm{CI},-1.844,-0.547)$, but semen volume and all reproductive hormones were not markedly altered.

Conclusion: Poor sleep quality might be related to impaired semen quality, but we found no evidence that poor sleep quality affects reproductive hormones.

Keywords: sleep quality, fertility, male reproduction, sperm quality, PSQI

\section{Introduction}

Bedtime and sleep quality have significant effects on human health. In the modern era, humans have been reported to sleep for fewer hours (1-2 hrs) per night than their ancestors. ${ }^{1}$ Consequently, the incidence of sleep disorders has progressively 
increased, being among the frequently encountered health problems by clinicians. ${ }^{2}$ In the general population, more than half of the adults have intermittent sleep disorders, and $15-20 \%$ have a chronic sleep problem. ${ }^{3}$ Previous studies reported that both restricted and excessive sleep duration are associated with obesity, cardiovascular disease, and all-cause mortality. ${ }^{4-6}$

Infertility is considered a public health epidemic. ${ }^{7,8}$ The World Health Organization (WHO) estimates that $9 \%$ of couples worldwide face fertility issues and that male factor contributes to $50 \%$ of the issues. ${ }^{9,10}$ Therefore, the effect of sleep on male fertility has attracted much attention in recent years. ${ }^{11}$ A recent animal study demonstrated that restricted sleep duration could significantly decrease sperm quality, ${ }^{12-14}$ and short sleep duration has also been associated with reduced fecundability in humans. ${ }^{15-17}$ In addition, Chen et $\mathrm{al}^{18}$ reported that sleep duration had an inverted U-shaped correlation pattern with both semen volume and total sperm count.

However, it still remains to be seen whether sleep disturbances are implicated in male infertility. Moreover, most epidemiologic studies have focused on the effects of sleep duration, rather than sleep quality. Thus, this study investigated the effects of sleep quality on sperm quality and reproductive hormones.

\section{Materials and Methods}

\section{Sample}

The volunteers were outpatients who were attending the Reproductive Medicine Center for various diagnostic purposes. A total of 1536 volunteers were examined from October 2017 to July 2019, and included in the present study. Among them, 566 were excluded from the analysis due to the following reasons: 135 had reproductive disorders (e.g. varicocele, hydrocele, cryptorchidism, orchitis, testicular torsion) or other chronic diseases (e.g. diabetes, uremia, cirrhosis of the liver, hypertension); 205 had undergone semen examination before; 107 reported a duration of abstinence of $<2$ days or $>7$ days; 32 had sample spillage or failed to collect the semen samples; 71 had been diagnosed with azoospermia or cryptozoospermia; and 16 had been diagnosed with neurological or psychiatric disorders. All the volunteers agreed to participate in this study, and signed an informed consent form. This study was approved by the Research Ethic Committee of the First Affiliated Hospital, College of Medicine, Zhejiang University
(No. 2017-708). The study was conducted in accordance with the principles of the Declaration of Helsinki.

\section{Questionnaire}

The participants responded to questions concerning the demographics (i.e. age, education and occupation) and lifestyle (such as the frequency of intercourse, smoking, alcohol and coffee consumptions, exercise, and hot bath or sauna). They also disclosed their medical history, including a history of chronic diseases, urologic or reproductive system diseases, neurological or psychiatric disorders, and recent fever $\left(\geq 38^{\circ} \mathrm{C}\right)$ in the last 3 months.

Sleep quality was measured using the Pittsburgh Sleep Quality Index (PSQI). ${ }^{19}$ This self-report index consists of 19 items that reflect respondents' sleep quality during the previous month. These items were grouped into seven components of sleep quality, namely: subjective sleep quality, sleep latency, sleep duration, habitual sleep efficiency, sleep disturbances, use of sleeping medication, and daytime dysfunction. Each component was scored at a $0-3$ interval scale with the overall PSQI scores ranging from 0 to 21 .

\section{Physical Examination}

The patients underwent a physical examination which was aimed at obtaining basic biological information, such as blood pressure, height, weight, and body mass index (BMI). In addition, secondary sexual characteristics (prominentia laryngea, breast, and penis), pudendal status, presence and volume of the testis (measured using a Prader orchidometer), status of the epididymis and the presence of varicocele were also evaluated.

\section{Semen Analysis}

Semen was obtained through masturbation after 2-7 days of abstinence. Each sample was collected into a sterile plastic container and taken to the laboratory within 30 mins after ejaculation. After liquefaction at $37^{\circ} \mathrm{C}$, semen samples were analyzed according to the WHO manual. The semen volume was measured with an Electronic Balance (BSA224S, Sartorius). Sperm concentration and progressive motility were evaluated in a Makler counting chamber (Sefi-Medical Instruments, Haifa, Israel). Normal sperm morphology was evaluated in samples stained using the Papanicolaou technique (Baso Diagnostics Inc., Zhuhai, China) and analyzed according to the WHO 2010 criteria. $^{20}$ 


\section{Serum Reproductive Hormones Determination}

On the morning of the examination, a blood sample was drawn from the cubital vein, centrifuged to obtain serum. Reproductive hormones (follicle-stimulating hormone, luteinizing hormone, estrogen, testosterone, and prolactin) were measured at the clinical laboratory of The First Affiliated Hospital, College of Medicine, Zhejiang University (Hangzhou, China) using the Beckman Access 2 Immunoassay System (Beckman Coulter Inc., California, US).

\section{Statistical Analysis}

We included the results of routine semen analysis, sperm morphology, and reproductive hormones analysis. Categorical variables were presented, as frequencies and percentages while continuous variables were presented as median (25-75 percentiles). The participants were divided into the good sleep and the poor sleep groups based on a previously validated ${ }^{21-23}$ cut-off point $(P S Q I=5)$. The total clinical score $\geq 5$ points indicate that the sleep quality of patients is poor. PSQI diagnostic specificity and sensitivity are $86.5 \%$ and $89.6 \%$, respectively. ${ }^{19,22}$ We assessed the relationship between sleep quality and potential confounders using chi-square test for categorical variables, and Kruskal-Wallis test for continuous variables.

We used multivariate linear regression analysis to examine the relationship between sleep quality and sperm parameters, reproductive hormones. Sleep quality was classified both as discrete variable (general PSQI scores) and dichotomous variable (PQSI $<5$ vs PQSI $\geq 5)$. Distributions of three semen parameters (volume, concentration, and total sperm number) were skewed; therefore we transformed them using the natural logarithm transformation to attain normal distribution before analysis. We created two models: an unadjusted model with only the sleep quality; and a second model with the main effects adjusted for potential confounders such as age, BMI, smoking, alcohol drinking, and ejaculation abstinence period, that were associated semen parameters. Spearman correlation coefficients were determined for general PSQI scores and all semen parameters.

Statistical significance was set at $P<0.05$ and statistical analyses were performed using the Statistical Package for the Social Sciences version 23.0 (SPSS, Inc., IBM).

\section{Results}

The demographic characteristics are shown in Table 1. The

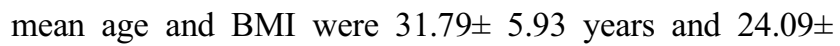
$3.65 \mathrm{~kg} / \mathrm{m}^{2}$, respectively, for the 970 participants. The median PSQI score was 4 (interquartile range, 3-6) and the mean PSQI score was $4.35 \pm 2.70$. The average length of sleep was $7.36 \pm 0.98 \mathrm{hrs}$. A total of 378 participants (38.97\%) were classified into poor sleep (general PSQI scores $\geq 5$ ). Compared with the good sleep group, they had an unhealthier lifestyle, higher BMI, more often consumed alcohol and smoked, and did less physical activity. In addition, they had a lower frequency of intercourse $(5.34 \pm 3.23$ per month) and shorter duration of sleep $(6.86 \pm 1.02 \mathrm{hrs})$.

Table 2 shows the unadjusted semen parameters and reproductive hormones. The results of semen parameters and reproductive hormone parameters were represented by the median (25-75 percentiles). The Kruskal-Wallis test showed that the median value of total motility, progressive motility, concentration, total sperm number, and normal sperm morphology in the poor sleep group was significantly lower than those in the good sleep group ( $p<0.001)$ (Figure 1). There were no statistically significant differences in semen volume and all reproductive hormones between the two groups.

Spearman correlation coefficients were determined for the general PSQI scores and all semen parameters (Figure 2). Total motility $(\mathrm{r}=-0.187979, p<0.001)$, progressive motility $(\mathrm{r}=-0.192902, p<0.001)$, concentration $(\mathrm{r}=-0.167063$, $p<0.001)$, total sperm number $(\mathrm{r}=-0.160008, p<0.001)$, and normal sperm morphology $(\mathrm{r}=-0.124511, p<0.001)$ were negatively correlated to the general PSQI scores. There was, however, no significant correlation between the semen volume, all reproductive hormones and the general PSQI scores.

Regression analysis is shown in Table 3. In unadjusted analyses, the general PSQI scores were significantly associated with total motility, progressive motility, concentration, total sperm number, and normal sperm morphology, but not with semen volume. These associations remained significant after adjusting for age, BMI, smoking, alcohol consumption, and ejaculation abstinence period, however, the associations were somewhat attenuated. In addition, we observed an inverse association between poor sleep group (PSQI $\geq 5$ ) and the semen parameters, both before and after the adjustments.

\section{Discussion}

In this study involving 970 outpatients from the Reproductive Medicine Center, total motility, progressive 
Table I Demographic Characteristics of Study Sample

\begin{tabular}{|c|c|c|c|c|}
\hline Characteristics & Total $(n=970)$ & Good Sleep $(n=592)$ & Poor Sleep $(n=378)$ & $p$-value ${ }^{a}$ \\
\hline Age (year) ${ }^{b}$ & $31.79 \pm 5.93$ & $31.90 \pm 5.97$ & $31.63 \pm 5.87$ & 0.630 \\
\hline BMI $\left(\mathrm{kg} / \mathrm{m}^{2}\right)^{\mathrm{b}}$ & $24.09 \pm 3.65$ & $23.76 \pm 3.36$ & $24.60 \pm 4.02$ & 0.006 \\
\hline Frequency of intercourse & $5.80 \pm 3.35$ & $6.10 \pm 3.40$ & $5.34 \pm 3.23$ & $<0.001$ \\
\hline (per month) ${ }^{b}$ & & & & \\
\hline Education, n (\%) & & & & 0.472 \\
\hline Less than college & $407(41.96)$ & $243(4 I .05)$ & I64(43.39) & \\
\hline College and higher & $563(58.04)$ & $349(58.95)$ & $214(56.61)$ & \\
\hline Employed n (\%) & & & & 0.167 \\
\hline Yes & $904(93.20)$ & $557(94.09)$ & $347(91.80)$ & \\
\hline No & $66(6.80)$ & $35(5.91)$ & $31(8.20)$ & \\
\hline Smoking, n (\%) & & & & 0.022 \\
\hline No & $660(68.04)$ & $419(70.78)$ & $24 I(63.76)$ & \\
\hline Yes & $310(31.96)$ & $173(29.22)$ & $137(36.24)$ & \\
\hline Alcohol, n (\%) & & & & 0.012 \\
\hline No & 785(80.93) & 494(83.45) & $291(76.98)$ & \\
\hline Yes & $185(19.07)$ & $98(16.55)$ & $87(23.02)$ & \\
\hline Coffee, n (\%) & & & & 0.661 \\
\hline No & $875(90.21)$ & $536(90.54)$ & $339(89.68)$ & \\
\hline Yes & $95(9.79)$ & $56(9.46)$ & $39(10.32)$ & \\
\hline Physically activity, n (\%) & & & & 0.027 \\
\hline No & $663(68.35)$ & $389(65.7 I)$ & $275(72.75)$ & \\
\hline Yes & $307(31.65)$ & $203(34.29)$ & $104(27.25)$ & \\
\hline Fever n (\%) & & & & 0.242 \\
\hline No & $944(97.32)$ & $579(97.80)$ & $365(96.56)$ & \\
\hline Yes & $26(2.68)$ & $13(2.20)$ & $13(3.44)$ & \\
\hline Global PSQI score ${ }^{b}$ & $4.35 \pm 2.69$ & $2.68 \pm 1.24$ & $7.69 \pm 0.79$ & $<0.001$ \\
\hline Duration of sleep (hours) ${ }^{b}$ & $7.36 \pm 0.98$ & $7.69 \pm 0.79$ & $6.86 \pm 1.02$ & $<0.001$ \\
\hline Bedtime $^{\text {bc }}$ & $23.18 \pm 1.12$ & $23.03 \pm 1.03$ & $23.43 \pm 1.21$ & $<0.001$ \\
\hline
\end{tabular}

Notes: ${ }^{a}$ Comparison between groups: continuous variable (Kruskal-Wallis test), categorical variables $\left(\chi^{2}\right.$-test). ${ }^{b}$ Data are expressed as mean \pm standard deviation. ${ }^{\mathrm{c}}$ For analysis purposes, minutes were divided by 60 and multiplied by 100. To obtain a metric variable, hours were counted from 0 to 24 , and hours after midnight were counted as 25 (for 1:00), 26 (for 2:00), etc.

Abbreviations: BMI, body mass index; PSQI, Pittsburg Sleep Quality Index.

motility, concentration, total sperm number, and normal sperm morphology were found to be associated with sleep quality. However, there was no significant relationship between reproductive hormone and sleep quality. To the best of our knowledge, this is the first study to describe the associations of sleep quality with semen quality, using the Pittsburgh Sleep Quality Index.

To date, the underlying mechanisms of the association between sleep and male reproductive function are still unclear in humans. Very similar to our result, Jensen et al, Chen et al and Ruge et al ${ }^{18,24,25}$ did not find any association between sleep quality and reproductive hormones in their studies. However, Ruge et $\mathrm{al}^{25}$ found an association between excessive sleep and reduced concentrations of testosterone, while Jankowski et $\mathrm{al}^{26}$ found greater sleep loss was associated with lower levels of dehydroepiandrosterone (DHEA). Due to the inconsistent results of these studies, we suggest that this relationship could be affected by the circadian system, which has been established to be important in mature sperm production process. ${ }^{27-30}$ Inappropriate sleep habits disrupt the rhythmic expression of circadian genes, leading to adverse effects on the male reproductive system. ${ }^{31,32}$ Since the circadian sleep-wake cycle is regulated by melatonin, endogenous circadian rhythm disorders could disrupt the cycle by deregulating the production of melatonin 
Table 2 Semen Parameters and Reproductive Hormones in Each Group According to the Global PSQI Scores

\begin{tabular}{|c|c|c|c|c|}
\hline Semen Parameter & Total $(n=970)$ & Good Sleep $(n=592)$ & Poor Sleep $(n=378)$ & $p$-value \\
\hline Duration of abstinence (days) ${ }^{\mathrm{a}}$ & $4.22 \pm 2.42$ & $4.18 \pm 1.52$ & $4.28 \pm 1.62$ & 0.426 \\
\hline Volume (mL) & $3.3(2.4,4.3)$ & $3.3(2.4,4.4)$ & $3.2(2.4,4.3)$ & 0.965 \\
\hline Total motility (\%) & $61.0(42.0,73.0)$ & $64.0(50.0,75.0)$ & $53.5(32.0,71.0)$ & $<0.001$ \\
\hline Progressive motility (\%) & $57.0(39.0,70.0)$ & $60.0(46.3,71.0)$ & $50.0(29.0,67.0)$ & $<0.001$ \\
\hline Concentration $\left(10^{6} / \mathrm{mL}\right)$ & $45.8(25.5,76.3)$ & $49.8(29.0,83.3)$ & $39.1(18.5,65.7)$ & $<0.001$ \\
\hline Total sperm number ( $10^{6} /$ ejaculate $)$ & I45.0(74.I, 253.8) & 159.2(85.2, 27।.2) & $122.5(58.2,221.4)$ & $<0.001$ \\
\hline Normal sperm morphology (\%) & $9.6(6.0,13.4)$ & $10.0(6.5,13.6)$ & $9.0(4.9,12.9)$ & $<0.001$ \\
\hline $\mathrm{FSH}(\mathrm{mlU} / \mathrm{mL})$ & $4.69(3.57,6.24)$ & $4.69(3.62,6.32)$ & $4.61(3.40,6.58)$ & 0.987 \\
\hline $\mathrm{LH}(\mathrm{mlU} / \mathrm{mL})$ & $4.00(2.96,5.40)$ & $3.92(2.90,5.36)$ & $4.28(3.28,5.49)$ & 0.234 \\
\hline $\mathrm{E} 2(\mathrm{pg} / \mathrm{mL})$ & $35.00(26.00,47.00)$ & $34.00(25.00,46.50)$ & $35.50(26.00,46.50)$ & 0.694 \\
\hline $\mathrm{T}(\mathrm{ng} / \mathrm{mL})$ & $3.86(2.97,4.96)$ & $3.85(2.92,4.90)$ & $3.73(3.03,5.04)$ & 0.956 \\
\hline PRL (ng/mL) & $8.74(6.4 \mathrm{I}, \mathrm{II} .9 \mathrm{I})$ & $8.64(6.18,11.50)$ & $9.07(7.23,12.36)$ & 0.107 \\
\hline
\end{tabular}

Notes: Data are presented as median (25-75 percentiles), comparison between each group: Kruskal-Wallis test. ${ }^{a}$ Data are expressed as mean \pm standard deviation. Abbreviations: $\mathrm{FSH}$, follicle-stimulating hormone; LH, luteinizing hormone; E2, estrogen; T, testosterone; PRL prolactin.

and cortisol. ${ }^{33}$ Melatonin, in turn, would affect the secretion of gonadotropin and testosterone, which promotes testicular maturation. ${ }^{34}$ A previous study showed that the melatonin was higher in workers without night shift, than those with night shift. ${ }^{35}$ Another study ${ }^{36}$ reported that the melatonin level in urine was positively correlated with sperm concentration. Consistent with these two studies, our findings demonstrated that melatonin and circadian system play an important role in determining male reproduction. In addition, we suspect that the mechanism may also be associated with lifestyle factors, since the men with poor sleep had higher BMI, lesser physical activity and higher frequencies of smoking and drinking. Even after adjusting for BMI, smoking, drinking, physical activity, and frequency of intercourse, the association between sleep quality and semen parameters remained significant.

Our results revealed a significant connection between sleep and sperm quality, and were consistent with previous studies. We observed that participants with poor sleep quality, also slept late and had decreased sperm parameters, suggesting that staying up late could be an important factor in reducing fertility. ${ }^{17}$ In addition, we found that people with poor sleep quality had shorter sleep duration and lower sperm quality. Similarly, a recent study ${ }^{15}$ found that both short sleep duration and long sleep duration were associated with decreased fertility, although the association between long sleep duration and decreased fertility was weak and inaccurate. However, the relationship between sleep disturbances, sleep duration and semen quality is still a controversial topic. Previous studies had shown an inverse U-shaped relationship, ${ }^{16,18,24}$ while our study found that most semen parameters declined as sleep quality decreased, and there was no evidence of a U-shaped relationship (Figure 2). This difference could be attributed to the difference in the type of participants studied. Our participants were recruited from fertility clinics, while theirs were from colleges or enlisted military.

This study had a number of advantages. First, we used PSQI, a self-report index consisting of 19 items that reflects respondents' sleep quality, to measure subjective sleep quality. Traditional sleep assessments only take into account the length of sleep. Second, the respondents had no prior knowledge of their fertility since they went to the hospital for the first time on the day of semen examination. Third, we examined many factors that could affect semen quality, such as BMI, smoking, alcohol, coffee, physical activity, and fever. The study had the following limitations. First, since this was a crosssectional study, the partners of participants were not followed up for pregnancy. Moreover, the quality of semen does not always correlate with fertility. Second, the participants were recruited from one fertility clinic rather than the general population, increasing the possibility of selection bias. Some participants had fertility stress, which could have led to decreased sleep quality. Finally, we used only one semen sample to predict male reproductive function.

\section{Conclusion}

In conclusion, we present evidence that poor sleep quality is correlated with lower total motility, progressive motility, concentration, total sperm count, and normal sperm 

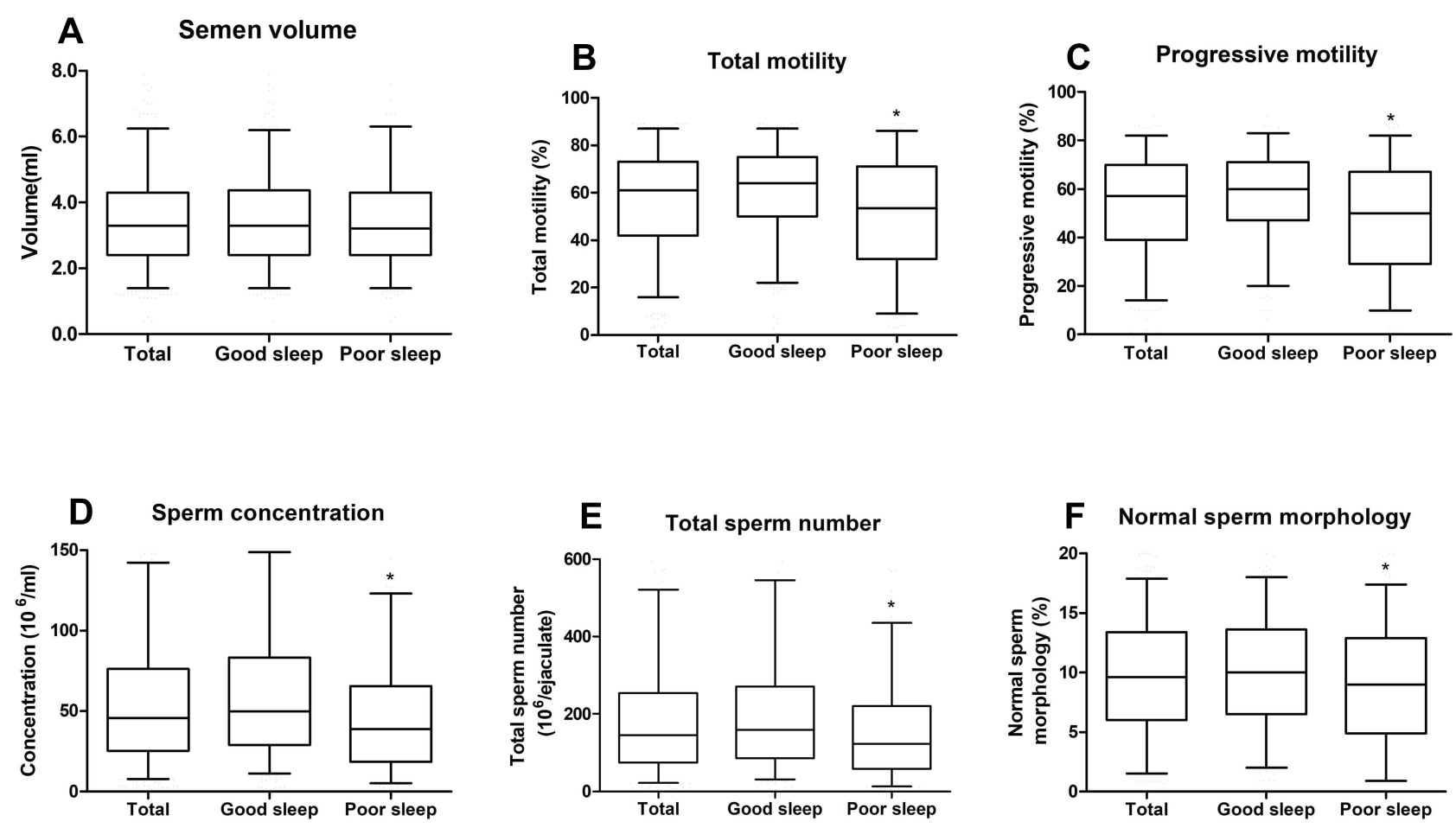

Figure I Boxplots showing various semen parameters grouped by PSQI among participants. The center horizontal line in each box represents the median, with the top and bottom edges of the box representing the 75th and 25th percentiles, respectively. This figure shows various routine semen parameters (A) semen volume, (B) total motility, (C) progressive motility, (D) concentration, (E) total sperm number, (F) normal sperm morphology). ${ }^{*} p<0.00$ I vs Good sleep.
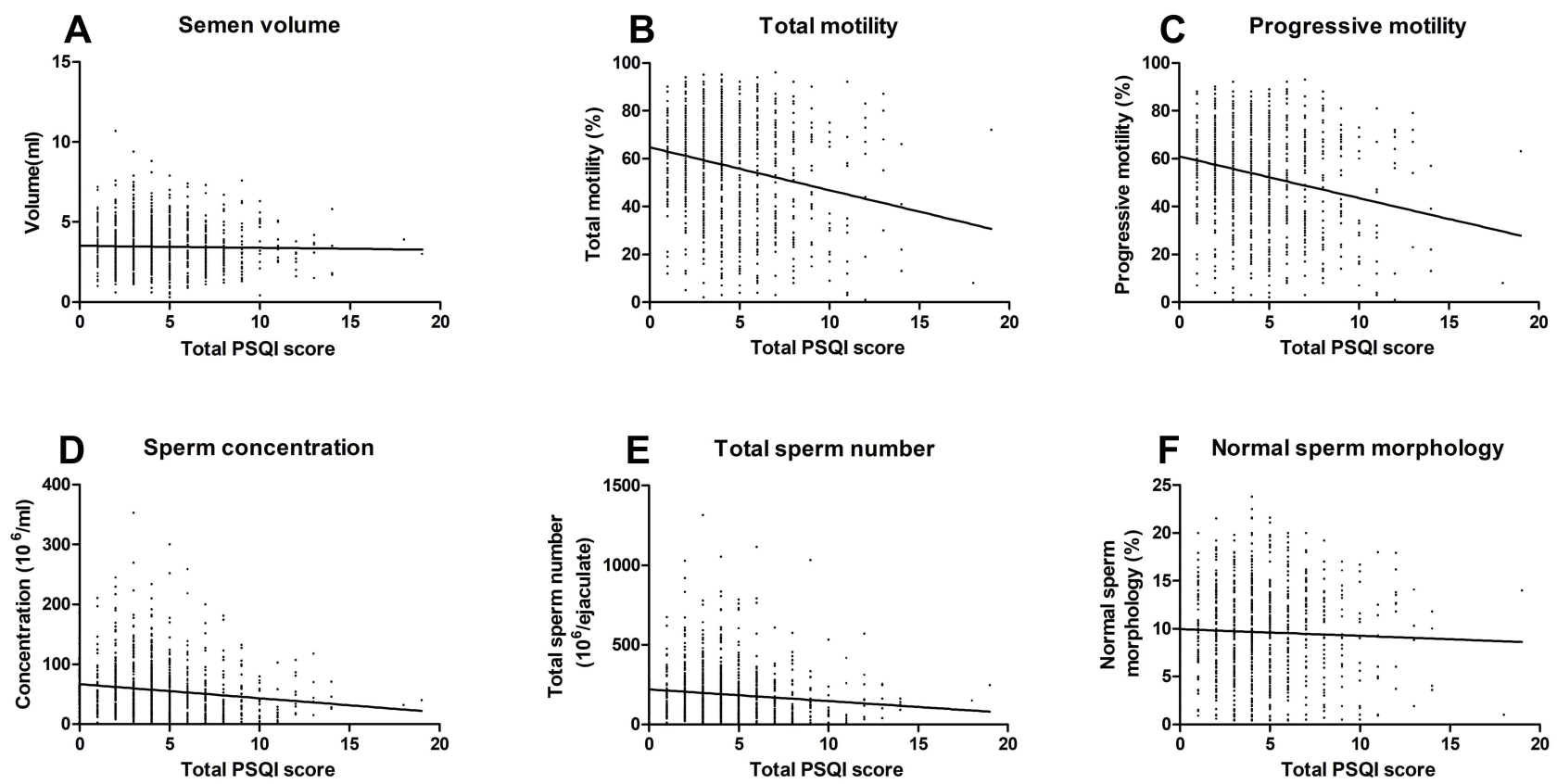

Figure 2 The total PSQI score and semen parameters. Spearman correlation analysis. Routine semen analysis. $(\mathbf{A})$ semen volume $(r=-0.004 \mid 32$, $p=0.898) ;(B)$ total motility ( $r=-0.187979, p<0.001)$; $(\mathbf{C})$ progressive motility $(r=-0.192902, p<0.001) ;(D)$ concentration $(r=-0.167063, p<0.001) ;(E)$ total sperm number $(r=-0.160008$, $p<0.001)$; (F) normal sperm morphology $(r=-0.124511, p<0.001)$

morphology. This indicates that good sleep quality may predict better reproductive potential in men of childbearing age. However, we found no association between sleep quality and reproductive hormones. Thus, further research is needed to clarify the mechanism underlying the relationship between sleep and male fertility. 


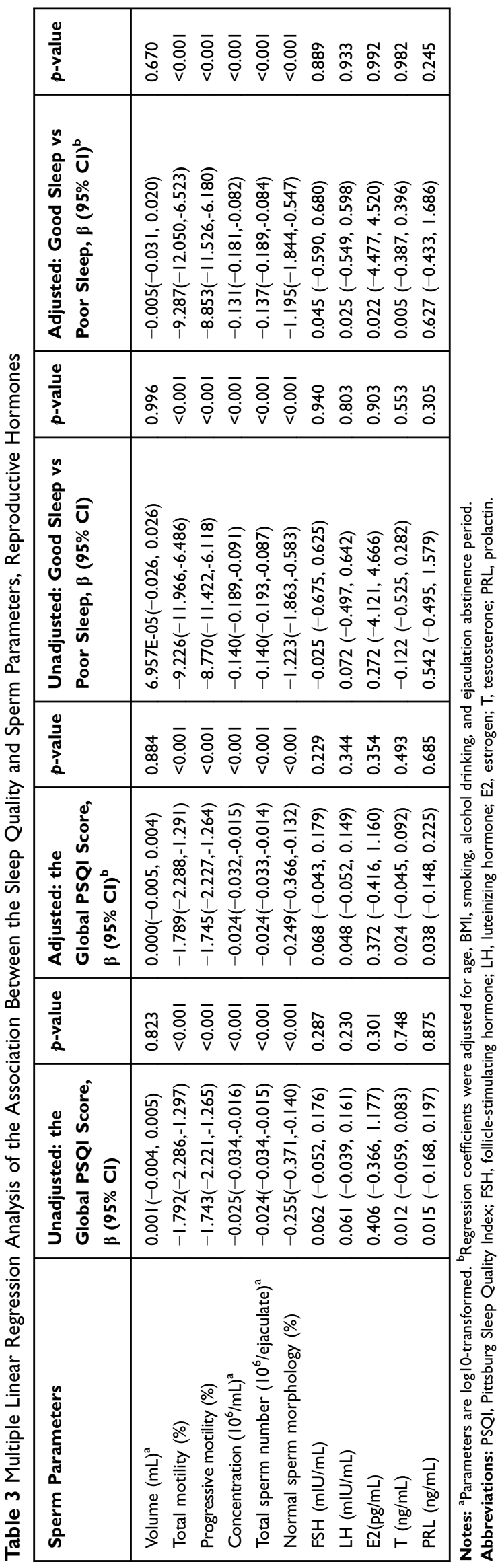

\section{Acknowledgment}

The authors are grateful to colleagues from Reproductive Medicine Center of The First Affiliated Hospital, College of Medicine, Zhejiang University for providing direct assistance and constructive suggestions for this research.

\section{Author Contributions}

All authors contributed to study concept and design. CongQi Du performed the statistical analyses and drafted the manuscript. All authors interpreted the data and critically revised the manuscript, approved the final version of the manuscript, and agreed to be accountable for all aspects of the work.

\section{Disclosure}

The authors declare no potential conflicts of interest with respect to the research, authorship and publication of this article.

\section{References}

1. Roenneberg T. Chronobiology: the human sleep project. Nature. 2013;498(7455):427-428. doi:10.1038/498427a

2. Mollayeva T, Thurairajah P, Burton K, Mollayeva S, Shapiro CM, Colantonio A. The Pittsburgh sleep quality index as a screening tool for sleep dysfunction in clinical and non-clinical samples: a systematic review and meta-analysis. Sleep Med Rev. 2016;25:52-73. doi:10.1016/j.smrv.2015.01.009

3. Ford ES, Cunningham TJ, Croft JB. Trends in self-reported sleep duration among US adults from 1985 to 2012. Sleep. 2015;38 (5):829-832. doi:10.5665/sleep.4684

4. Rod NH, Vahtera J, Westerlund H, et al. Sleep disturbances and cause-specific mortality: results from the GAZEL cohort study. Am J Epidemiol. 2011;173(3):300-309. doi:10.1093/aje/kwq371

5. Cepeda MS, Stang P, Blacketer C, Kent JM, Wittenberg GM. Clinical relevance of sleep duration: results from a cross-sectional analysis using NHANES. J Clin Sleep Med. 2016;12(6):813-819. doi:10. $5664 / \mathrm{jcsm} .5876$

6. Theorell-Haglow J, Lindberg E. Sleep duration and obesity in adults: what are the connections? Curr Obes Rep. 2016;5(3):333-343. doi:10.1007/s13679-016-0225-8

7. Irvine DS. Epidemiology and aetiology of male infertility. Hum Reprod. 1998;13(Suppl 1):33-44. doi:10.1093/humrep/13.suppl_1.33

8. Tournaye H, Krausz C, Oates RD. Novel concepts in the aetiology of male reproductive impairment. Lancet Diabetes Endocrinol. 2017;5 (7):544-553. doi:10.1016/S2213-8587(16)30040-7

9. Boivin J, Bunting L, Collins JA, Nygren KG. International estimates of infertility prevalence and treatment-seeking: potential need and demand for infertility medical care. Hum Reprod. 2007;22 (6):1506-1512. doi:10.1093/humrep/dem046

10. Agarwal A, Mulgund A, Hamada A, Chyatte MR. A unique view on male infertility around the globe. Reprod Biol Endocrinol. 2015;13:37. doi:10.1186/s12958-015-0032-1

11. Palnitkar G, Phillips CL, Hoyos CM, Marren AJ, Bowman MC, Yee BJ. Linking sleep disturbance to idiopathic male infertility. Sleep Med Rev. 2018;42:149-159. doi:10.1016/j.smrv.2018.07.006

12. Alvarenga TA, Hirotsu C, Mazaro-Costa R, Tufik S, Andersen ML. Impairment of male reproductive function after sleep deprivation. Fertil Steril. 2015;103(5):1355-1362 e1351. doi:10.1016/j.fertnstert. 2015.02.002 
13. Choi JH, Lee SH, Bae JH, et al. Effect of sleep deprivation on the male reproductive system in rats. J Korean Med Sci. 2016;31 (10):1624-1630. doi:10.3346/jkms.2016.31.10.1624

14. Torres M, Laguna-Barraza R, Dalmases M, et al. Male fertility is reduced by chronic intermittent hypoxia mimicking sleep apnea in mice. Sleep. 2014;37(11):1757-1765. doi:10.5665/sleep. 4166

15. Wise LA, Rothman KJ, Wesselink AK, et al. Male sleep duration and fecundability in a North American preconception cohort study. Fertil Steril. 2018;109(3):453-459. doi:10.1016/j.fertnstert.2017. 11.037

16. Wang X, Chen Q, Zou P, et al. Sleep duration is associated with sperm chromatin integrity among young men in Chongqing, China. J Sleep Res. 2018;27(4):e12615. doi:10.1111/jsr.2018.27. issue-4

17. Liu MM, Liu L, Chen L, et al. Sleep deprivation and late bedtime impair sperm health through increasing antisperm antibody production: a prospective study of 981 healthy men. Med Sci Monit. 2017;23:1842-1848. doi:10.12659/MSM.900101

18. Chen Q, Yang H, Zhou N, et al. Inverse U-shaped association between sleep duration and semen quality: longitudinal observational study (MARHCS) in Chongqing, China. Sleep. 2016;39(1):79-86. doi: $10.5665 /$ sleep. 5322

19. Buysse DJ, Reynolds CF 3rd, Monk TH, Berman SR, Kupfer DJ. The Pittsburgh Sleep Quality Index: a new instrument for psychiatric practice and research. Psychiatry Res. 1989;28(2):193-213. doi:10.1016/0165-1781(89)90047-4

20. Mao H, Feng L, Yang WX. Environmental factors contributed to circannual rhythm of semen quality. Chronobiol Int. 2017;34 (3):411-425. doi:10.1080/07420528.2017.1280046

21. Akman T, Yavuzsen T, Sevgen Z, Ellidokuz H, Yilmaz AU. Evaluation of sleep disorders in cancer patients based on Pittsburgh Sleep Quality Index. Eur J Cancer Care (Engl). 2015;24(4):553-559. doi:10.1111/ecc.2015.24.issue-4

22. Buysse DJ, Reynolds CF 3rd, Monk TH, Hoch CC, Yeager AL, Kupfer DJ. Quantification of subjective sleep quality in healthy elderly men and women using the Pittsburgh Sleep Quality Index (PSQI). Sleep. 1991;14(4):331-338.

23. Ozkan SA, Rathfisch G. The effect of relaxation exercises on sleep quality in pregnant women in the third trimester: a randomized controlled trial. Complement Ther Clin Pract. 2018;32:79-84. doi:10. 1016/j.ctcp.2018.05.008
24. Jensen TK, Andersson AM, Skakkebaek NE, et al. Association of sleep disturbances with reduced semen quality: a cross-sectional study among 953 healthy young Danish men. Am J Epidemiol. 2013;177(10):1027-1037. doi:10.1093/aje/kws420

25. Ruge M, Skaaby T, Andersson AM, Linneberg A. Cross-sectional analysis of sleep hours and quality with sex hormones in men. Endocr Connections. 2019;141-149. doi:10.1530/EC-18-0548

26. Jankowski KS, Fajkowska M, Domaradzka E, Wytykowska A. Chronotype, social jetlag and sleep loss in relation to sex steroids. Psychoneuroendocrinology. 2019;108:87-93. doi:10.1016/j.psyneuen. 2019.05.027

27. Alvarez JD, Hansen A, Ord T, et al. The circadian clock protein BMAL1 is necessary for fertility and proper testosterone production in mice. J Biol Rhythms. 2008;23(1):26-36. doi:10.1177/0748730407311254

28. Beaver LM, Gvakharia BO, Vollintine TS, Hege DM, Stanewsky R, Giebultowicz JM. Loss of circadian clock function decreases reproductive fitness in males of Drosophila melanogaster. Proc Natl Acad Sci U S A. 2002;99(4):2134-2139. doi:10.1073/pnas.032426699

29. Tobback J, Boerjan B, Vandersmissen HP, Huybrechts R. Male reproduction is affected by RNA interference of period and timeless in the desert locust Schistocerca gregaria. Insect Biochem Mol Biol. 2012;42(2):109-115. doi:10.1016/j.ibmb.2011.11.003

30. Peterlin A, Kunej T, Peterlin B. The role of circadian rhythm in male reproduction. Curr Opin Endocrinol Diabetes Obes. 2019;26 (6):313-316. doi:10.1097/MED.0000000000000512

31. Boden MJ, Varcoe TJ, Kennaway DJ. Circadian regulation of reproduction: from gamete to offspring. Prog Biophys Mol Biol. 2013;113 (3):387-397. doi:10.1016/j.pbiomolbio.2013.01.003

32. Gamble KL, Resuehr D, Johnson CH. Shift work and circadian dysregulation of reproduction. Front Endocrinol (Lausanne). 2013;4:92. doi:10.3389/fendo.2013.00092

33. Eckel-Mahan K, Sassone-Corsi P. Metabolism and the circadian clock converge. Physiol Rev. 2013;93(1):107-135. doi:10.1152/ physrev.00016.2012

34. Li C, Zhou X. Melatonin and male reproduction. Clin Chim Acta. 2015;446:175-180. doi:10.1016/j.cca.2015.04.029

35. Bhatti P, Mirick DK, Randolph TW, et al. Oxidative DNA damage during night shift work. Occup Environ Med. 2017;74(9):680-683. doi:10.1136/oemed-2017-104414

36. Ortiz A, Espino J, Bejarano I, et al. High endogenous melatonin concentrations enhance sperm quality and short-term in vitro exposure to melatonin improves aspects of sperm motility. $J$ Pineal Res. 2011;50(2):132-139. doi:10.1111/j.1600-079X.2010.00822.x
Nature and Science of Sleep

\section{Publish your work in this journal}

Nature and Science of Sleep is an international, peer-reviewed, open access journal covering all aspects of sleep science and sleep medicine, including the neurophysiology and functions of sleep, the genetics of sleep, sleep and society, biological rhythms, dreaming, sleep disorders and therapy, and strategies to optimize healthy sleep.
The manuscript management system is completely online and includes a very quick and fair peer-review system, which is all easy to use. Visit http://www.dovepress.com/testimonials.php to read real quotes from published authors. 